EPJ manuscript No.

(will be inserted by the editor)

\title{
Preparation of a Stable and Maximally Entangled State of Two Distant Qutrits Trapped in Separate Cavities
}

Chang-shui Yu, X. X. Yi, He-shan Song ${ }^{\mathrm{a}}$, and D. Mei

School of Physics and Optoelectronic Technology, Dalian University of Technology, Dalian 116024, P. R. China

Received: date / Revised version: date

\begin{abstract}
We have proposed a simple scheme to entangle two distant qutrits trapped in separate optical cavities. The quantum information of each qutrit is skillfully encoded on the degenerate ground states of a pair of atoms, hence the entanglement between them is relatively stable against spontaneous emission. In Lamb-Dicke limits, it is not necessary to require coincidence detections, which will relax the conditions for the experimental realization. The scheme is robust against the inefficient detections.
\end{abstract}

PACS. 42.50.Dv 03.67.Mn - 03.65.Ud

Entanglement, in particular the entanglement between distant particles, is not only a key ingredient for the tests of quantum nonlocality [1], but also an important physical resource in achieving tasks of quantum computation and quantum communication [2]. Hence, generation of entangled states and its further applications are immensely important. A lot of schemes have been proposed to generate entangled states [3-9], but most of the schemes focused on the generation of entanglement of two qubits or more qubits. However the entanglement between two qubits (E.g. EPR pairs) and even many qubits (For example, GHZ or W states) can not be competent for all the

\footnotetext{
${ }^{a}$ Email address:hssong@dlut.edu.cn
}

tasks of quantum information processing. In particular, higher-dimensional entanglement has recently attracted increasing interests: A maximally entangled state of two qudits is necessary in general, if an unknown quantum state of qudit will be teleported exactly [10]; A known quantum state can not be remotely prepared, unless an entangled state of two qudits has been provided [11,12]; Moreover, cryptographic protocols based on entangled qutrits [13-16] have been shown to be more efficient and secure than those based on qubit systems; Recently Ref. [17] has shown that teleportation can also be implemented in faith, 
even though a non-maximally entangled states defined in higher dimension can be provided.

Based on the demand of entangled qutrits, there have been some schemes [18-22] to generate entangled qutrits. However, these schemes are mainly focused on the preparation of entangled qutrits at the confined location (for example, both are trapped in a single cavity) and the generation of entangled photons of qutrits. In particular, the partial quantum information of a qutrit is encoded on the excited state of atoms (ions) in some schemes, which means that the entanglement of the qutrits is fragile (not stable). So far as we know, few schemes have been found to effectively entangle distant atoms or ions of qutrits. In this paper, we propose a simple scheme to generate stable maximally entangled state of two distant qutrits with the help of linear optical elements. It should first be worth noting that, although we will employ the same atom-cavity interaction mechanism as our previous work [23], it is not a simple extension but we address a new physical problem by a creative design of quantum optical circuits. In our scheme, the most important is that the quantum information of each qutrit is skillfully encoded on the degenerate ground states of a pair of separate atoms trapped in optical cavities, respectively, which leads to a relatively stable entangled state. In addition, The key of the scheme is the indistinguishability of photons emitting from the entangled atoms, which has been widely employed to entangle distant qubits [24-29]. Our scheme is shown to be robust against the inefficient detections. In particular, in LambDicke limits, we do not require the coincidence detections of photons, which can dramatically relax the conditions of practical realization.

The systems we consider here are the same to those in Ref. [24] where two atoms trapped in separate cavities can be robustly entangled by simultaneously detecting the leakage photons. Here, we will show that the expanded version can help us to entangle two distant qutrits without coincidence detections. We consider two pairs of identical three-level $\Lambda$-type atoms trapped in four separated identical one-sided optical cavities $A_{1}, A_{2}, B_{1}$ and $B_{2}$, respectively, with cavities $A_{i}$ at Alice's side and cavities $B_{i}$ at Bob's. See FIG. 1 (a). Each atom has an excited state $|e\rangle$ and two degenerate ground states $\left|g_{l}\right\rangle$ and $\left|g_{r}\right\rangle$. The transitions $\left|g_{l}\right\rangle \rightarrow|e\rangle$ and $\left|g_{r}\right\rangle \rightarrow|e\rangle$ are strongly coupled to left- and right- circularly polarizing cavity modes, respectively. Our atomic level structure can be achieved by Zeeman sublevels [33] and has been realized to entangle two atoms [34]. The quantum information of a qutrit is encoded in the ground states of a pair of atoms as follows.

$$
\begin{aligned}
& |0\rangle_{\beta}=\left|g_{l} g_{l}\right\rangle_{\beta},|2\rangle_{\beta}=\left|g_{r} g_{r}\right\rangle_{\beta}, \\
& |1\rangle_{\beta}=\frac{1}{\sqrt{2}}\left(\left|g_{l} g_{r}\right\rangle_{\beta}+\left|g_{r} g_{l}\right\rangle_{\beta}\right),
\end{aligned}
$$

with $\beta=A, B$ denoting Alice or Bob. The experimental setup is sketched in FIG. 1 (b). We suppose the four atoms are all initially prepared in their excited states and cavities in the vacuum state. If all the four detectors are clicked, Alice and Bob can share a stable and maximally entangled bipartite quantum state of qutrits:

$$
|\Psi\rangle_{A B}=\frac{1}{\sqrt{3}}\left(\left|0_{A} 0_{B}\right\rangle+\left|1_{A} 1_{B}\right\rangle+\left|2_{A} 2_{B}\right\rangle\right) .
$$


In order to demonstrate our scheme in detail, let us start with the interaction between atoms and cavities. The Hamiltonian governing the evolution of the four atomcavity systems can be given in the interaction picture by (setting $\hbar=1)$

$$
\begin{aligned}
H_{j} & =\lambda_{L}|e\rangle_{j j}\left\langle g_{l}\left|a_{L}^{j}+\lambda_{R}\right| e\right\rangle_{j j}\left\langle g_{r}\right| a_{R}^{j}+\text { h.c. }, \\
j & =A_{\alpha}, B_{\alpha}, \alpha=1,2,
\end{aligned}
$$

where $L, R$ denote the left- and right- circularly polarizing cavity modes, $a_{k}^{j \dagger}, a_{k}^{j}$ are the creation and annihilation operators of the $k$ mode in the $j$ cavity and $\lambda_{k}$ is the coupling constant. The upper levels $|e\rangle$ can decay to the two degenerate ground states $\left|g_{l}\right\rangle$ and $\left|g_{r}\right\rangle$ with the rates $2 \gamma_{l}$ and $2 \gamma_{r}$, respectively, and every cavity has a leakage rate $2 \kappa$. Hence, the master equation describing the evolution of density operator $\rho$ is given by

$$
\begin{gathered}
\dot{\rho}_{j}=-i\left(H_{e f f} \rho_{j}-\rho_{j} H_{e f f}^{\dagger}\right)+2 \kappa \sum_{k=L, R} a_{k}^{j} \rho_{j} a_{k}^{j \dagger} \\
+2 \sum_{p=l, r} \gamma_{p}\left|g_{p}\right\rangle_{j j}\left\langle e\left|\rho_{j}\right| e\right\rangle_{j j}\left\langle g_{p}\right|, j=A_{\alpha}, B_{\alpha}, \alpha=1,2,
\end{gathered}
$$

with

$$
H_{e f f}=H_{j}-i \kappa \sum_{k=L, R} a_{k}^{j \dagger} a_{k}^{j}-i\left(\gamma_{l}+\gamma_{r}\right)|e\rangle_{j j}\langle e|
$$

So long as spontaneous emissions do not happen and cavity photons are not leaked out, the above effective nonHermitian Hamiltonian (5) can be employed to describe the dynamics of the system based on quantum jump approach [30].

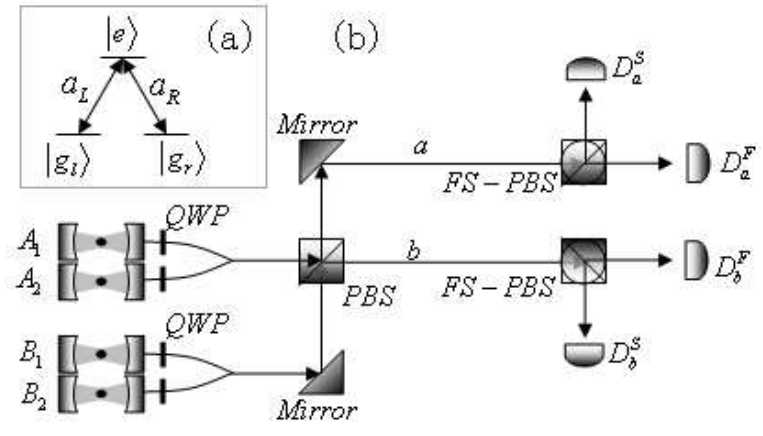

Fig. 1. (a) Atomic level structure. (b) Experimental setup for maximally entangled two qutrits without coincidence detections allowed. The pair of atoms trapped in cavities $A_{\alpha}, \alpha=$ 1,2 which are encoded by the quantum information of a qutrit are at Alice's location. The other pair of atoms in cavities $B_{\alpha}, \alpha=1,2$ which are encoded by another qutrit are at Bob's location. Circularly polarizing light becomes linearly polarizing by the quarter wave plates $(Q W P)$. Photons leaking out of cavities pass through the polarizing beam splitters $(P B S)$ which transmits $H$-polarizing photons and reflect $V$-polarizing photons. Before registered by the detectors, photons meet the rotated $P B S$ es $(F S-P B S)$ with the rotation angle $\theta$ which transmit $F$-polarizing photons and reflect $S$-polarizing photons.

Consider the initial state $|e\rangle_{j}|0\rangle_{j}$, after a time $t$ the state of each system will, respectively, become

$$
\begin{aligned}
|\psi(t)\rangle_{j} & =\frac{x|e\rangle_{j}|0\rangle_{j}+y\left|g_{l}\right\rangle_{j}|V\rangle_{j}+z\left|g_{r}\right\rangle_{j}|H\rangle_{j}}{\sqrt{|x|^{2}+|y|^{2}+|z|^{2}}}, \\
j & =A_{\alpha}, B_{\alpha}, \alpha=1,2
\end{aligned}
$$


where

$$
\begin{aligned}
& x=e^{-\frac{\Gamma}{2} t}\left[\cos \left(\Omega_{k} t\right)+\frac{\Delta}{2 \Omega_{k}} \sin \left(\Omega_{k} t\right)\right], \\
& y=-e^{-\frac{\Gamma}{2} t} \frac{i \sin \left(\Omega_{k} t\right)}{\Omega_{k}} \lambda_{L}, \\
& z=-e^{-\frac{\Gamma}{2} t} \frac{i \sin \left(\Omega_{k} t\right)}{\Omega_{k}} \lambda_{R},
\end{aligned}
$$

with

$$
\begin{aligned}
\Omega_{k} & =\sqrt{\Omega^{2}-\Delta^{2} / 4}, \\
\Omega^{2} & =\lambda_{L}^{2}+\lambda_{R}^{2}, \\
\Gamma & =\gamma_{l}+\gamma_{r}+\kappa, \\
\Delta & =\kappa-\gamma_{l}-\gamma_{r} .
\end{aligned}
$$

Here $|0\rangle_{j}$ and $|V\rangle_{j},|H\rangle_{j}$ present vacuum state and the one-photon state with vertically and horizontally polarizing cavity modes, respectively. From FIG. 1 (b), one can find that photons leaking out of cavities will pass through quarter wave plates $(Q W P)$ which change circularly polarizing light into linearly polarizing light. We assume leftand right- circularly polarizing photons become vertically $(V)$ and horizontally $(H)$ polarizing respectively [24]. In this sense, we have directly replaced the circularly polarizing photons in eq. (6) by linearly polarizing photons.

We have said that the spirit of our scheme is the indistinguishability of photons. This usually needs the coincidence detections of photons, otherwise the emission of a photon will lead to a recoil of the atom [25] which destroy the indistinguishability and lead to a failure. If our trapped atoms are restricted to operating in the LambDicke limit, where the recoil energy does not suffice to change the atomic motional state [25], the indistinguishability can be preserved. Hence in Lamb-Dicke limit we do not require the coincidence detections. Consequently we can suppose the evolution time of every subsystem to be $\tau_{j}$. In such an interval of time, one can obtain the state $\left|\psi\left(\tau_{j}\right)\right\rangle$ given by eq. (6) with the probability

$P_{j}=e^{-\Gamma \tau_{j}}\left\{\left[\cos \left(\Omega_{k} \tau_{j}\right)+\frac{\Delta}{2 \Omega_{k}} \sin \left(\Omega_{k} \tau_{j}\right)\right]^{2}+\frac{\sin ^{2}\left(\Omega_{k} \tau_{j}\right)}{\Omega_{k}^{2}} \Omega^{2}\right\}$.

Thus the joint state of the four systems can be given by $|\Psi\rangle=\otimes_{j}\left|\psi\left(\tau_{j}\right)\right\rangle$ with the probability $P_{1}=\prod_{j} P_{j}$. From eq. (6), one can find that the term $|e\rangle_{j}|0\rangle_{j}$ has no contribution to the detections, hence one can safely neglect it for simplification. As a result, one can rewrite eq. (6) at the time $\tau_{j}$ as

$$
\begin{aligned}
\left|\phi\left(\tau_{j}\right)\right\rangle_{j} & =\frac{1}{\Omega}\left(\lambda_{L}\left|g_{l}\right\rangle_{j}|V\rangle_{j}+\lambda_{R}\left|g_{r}\right\rangle_{j}|H\rangle_{j}\right), \\
j & =A_{\alpha}, B_{\alpha}, \alpha=1,2 .
\end{aligned}
$$

In the interval of $\max _{j}\left\{\tau_{j}\right\}$, the joint state of the whole four systems can be expressed by

$$
|\Phi\rangle=\otimes_{j} \frac{1}{\Omega}\left(\lambda_{L}\left|g_{l}\right\rangle_{j}|V\rangle_{j}+\lambda_{R}\left|g_{r}\right\rangle_{j}|H\rangle_{j}\right)
$$

with the total probability

$$
P_{2}=\prod_{j}\left[\frac{e^{-\Gamma \tau_{j}} \sin ^{2}\left(\Omega_{k} \tau_{j}\right) \Omega^{2}}{\Omega_{k}^{2}}\right] .
$$

Next we will show that the state $|\Phi\rangle$ can collapse to our desired state $|\Psi\rangle_{A B}$ given by eq. (2) in terms of noncoincidence detections in Lamb-Dicke limits. We assume that the four detectors are clicked at the different times which just correspond to a permutation of the evolution time $\left\{\tau_{j}\right\}$, where we neglect the transmission time through the linear optical elements. Because the photons leaking out of the cavities are indistinguishable in Lamb-Dicke 
limits, one can not tell which atom the photon emits from. In other words, one can not evaluate by which atom each click is led to. Therefore the evolution time $\left\{\tau_{j}\right\}$ is also indistinguishable for each atom. Let $t_{1} \leq t_{2} \leq t_{3} \leq t_{4}$ be the time sequence at which the detectors are clicked, and which corresponds to some permutation of $\left\{\tau_{j}\right\}$. Without loss of the generality, we suppose the detections are clicked in turn as $D_{a}^{F} \rightarrow D_{b}^{F} \rightarrow D_{a}^{S} \rightarrow D_{b}^{S}$ corresponding to the above time sequence. Following FIG. 1 (b). A photon leaking out of the cavity will first pass through a polarizing beam splitter $(P B S)$ which transmits $H$ polarizing light and reflects $V$-polarizing light and then meet a rotated $P B S(F S-P B S)$ which transforms $V$ and $H$-polarizing light as $|V\rangle \rightarrow \cos \theta|F\rangle+\sin \theta|S\rangle$ and $|H\rangle \rightarrow-\cos \theta|S\rangle+\sin \theta|F\rangle$ where $\theta$ is the rotated angle. $F S-P B S$ always transmits $F$-polarizing light and reflects $S$-polarizing light. Therefore if the detector $D_{a}^{F}$ is clicked at time $t_{1}$, the joint state will collapse to

$$
\begin{aligned}
& |\Phi\rangle_{1}=\frac{\cos \theta \lambda_{L}}{\Omega}\left(\left|g_{l}\right\rangle_{A_{1}}|\phi\rangle_{A_{2}}+|\phi\rangle_{A_{1}}\left|g_{l}\right\rangle_{A_{2}}\right)|\phi\rangle_{B_{1}}|\phi\rangle_{B_{2}} \\
& +\frac{\sin \theta \lambda_{R}}{\Omega}|\phi\rangle_{A_{1}}|\phi\rangle_{A_{2}}\left(\left|g_{r}\right\rangle_{B_{1}}|\phi\rangle_{B_{2}}+|\phi\rangle_{B_{1}}\left|g_{r}\right\rangle_{B_{2}}\right) .
\end{aligned}
$$

When detector $D_{b}^{F}$ is clicked at time $t_{2},|\Phi\rangle_{1}$ collapses to

$$
\begin{gathered}
|\Phi\rangle_{2}=\frac{1}{\Omega^{2}}\left[\sqrt{2} \cos \theta \sin \theta \lambda_{L} \lambda_{R}|1\rangle_{A}|\phi\rangle_{B_{1}}|\phi\rangle_{B_{2}}+\cos ^{2} \theta \lambda_{L}^{2}\right. \\
\times\left(\left|g_{l}\right\rangle_{A_{1}}|\phi\rangle_{A_{2}}+|\phi\rangle_{A_{1}}\left|g_{l}\right\rangle_{A_{2}}\right)\left(\left|g_{l}\right\rangle_{B_{1}}|\phi\rangle_{B_{2}}+|\phi\rangle_{B_{1}}\left|g_{l}\right\rangle_{B_{2}}\right) \\
\quad+\sin ^{2} \theta \lambda_{R}^{2}\left(\left|g_{r}\right\rangle_{A_{1}}|\phi\rangle_{A_{2}}+|\phi\rangle_{A_{1}}\left|g_{r}\right\rangle_{A_{2}}\right)\left(\left|g_{r}\right\rangle_{B_{1}}|\phi\rangle_{B_{2}}\right. \\
\left.\left.\quad+|\phi\rangle_{B_{1}}\left|g_{r}\right\rangle_{B_{2}}\right)+\sqrt{2} \sin \theta \cos \theta \lambda_{R} \lambda_{L}|\phi\rangle_{A_{1}}|\phi\rangle_{A_{2}}|1\rangle_{B}\right] .
\end{gathered}
$$

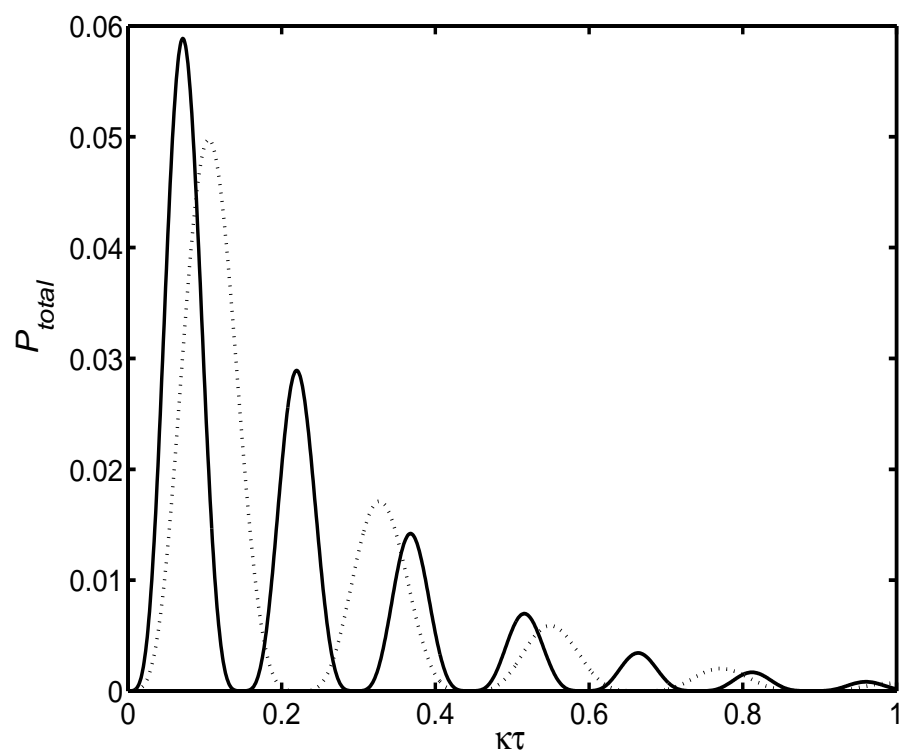

Fig. 2. (Dimensionless)The probability $P_{\text {total }}$ of getting a maximally entangled state of qutrits vs $\kappa \tau$ with different coupling constants. We choose $\tau_{j}=\tau, \gamma_{l}=\gamma_{r}=0.1 \kappa$ and $\lambda_{L}=\lambda_{R}=10 \kappa($ dotted line $)$ and $15 \kappa($ solid line $)$.

Analogously, if at time $t_{3}$ the detector $D_{a}^{S}$ is clicked, $|\Phi\rangle_{2}$ will collapse to $|\Phi\rangle_{3}$ as

$$
\begin{gathered}
|\Phi\rangle_{3}=\frac{1}{\Omega^{3}}\left[2 \cos ^{2} \theta \sin \theta \lambda_{L}^{3}|0\rangle_{A}\left(\left|g_{l}\right\rangle_{B_{1}}|\phi\rangle_{B_{2}}+|\phi\rangle_{B_{1}}\left|g_{l}\right\rangle_{B_{2}}\right)\right. \\
-\sqrt{2} \lambda_{L} \lambda_{R}^{2} \sin \theta \cos (2 \theta)|1\rangle_{A}\left(\left|g_{r}\right\rangle_{B_{1}}|\phi\rangle_{B_{2}}+|\phi\rangle_{B_{1}}\left|g_{r}\right\rangle_{B_{2}}\right) \\
-\sqrt{2} \lambda_{R} \lambda_{L}^{2} \cos \theta \cos (2 \theta)\left(\left|g_{l}\right\rangle_{A_{1}}|\phi\rangle_{A_{2}}+|\phi\rangle_{A_{1}}\left|g_{l}\right\rangle_{A_{2}}\right)|1\rangle_{B} \\
-2 \sin ^{2} \theta \cos \theta \lambda_{R}^{3}\left(\left|g_{r}\right\rangle_{A_{1}}|\phi\rangle_{A_{2}}+|\phi\rangle_{A_{1}}\left|g_{r}\right\rangle_{A_{2}}\right)|2\rangle_{B} .
\end{gathered}
$$

When the detector $D_{b}^{S}$ is clicked at time $t_{4},|\Phi\rangle_{3}$ collapses to

$$
\begin{aligned}
|\Phi\rangle_{4}= & \frac{1}{\Omega^{4}}\left(\sin ^{2}(2 \theta) \lambda_{L}^{4}|0\rangle_{A}|0\rangle_{B}+\sin ^{2}(2 \theta) \lambda_{R}^{4}|2\rangle_{A}|2\rangle_{B}\right. \\
& \left.+2 \cos ^{2}(2 \theta) \lambda_{L}^{2} \lambda_{R}^{2}|1\rangle_{A}|1\rangle_{B}\right) .
\end{aligned}
$$

Note that all the states $|\Phi\rangle_{m}, m=1,2,3,4$, are not normalized. It is obvious that if $\lambda_{L}=\lambda_{R}$ and $\tan ^{2}(2 \theta)=2$, one can obtain the desired state $|\Psi\rangle_{A B}$. In this case, the probability of getting $|\Psi\rangle_{A B}$ from $|\Phi\rangle$ (eq. (9)) can be given 
by

$$
P_{3}=\frac{2\left[\sin ^{4}(2 \theta)+2 \cos ^{4}(2 \theta)\right]}{16}=\frac{1}{12}
$$

Hence the total probability of getting the desired state is

$$
P_{\text {total }}=\frac{1}{12} \prod_{j}\left[\frac{e^{-\Gamma \tau_{j}} \sin ^{2}\left(\Omega_{k} \tau_{j}\right) \Omega^{2}}{\Omega_{k}^{2}}\right]
$$

In fact, it is worth noting that the scheme is deterministic because one can obtain the entangled state once he detects four photons. $P_{\text {total }}$ only corresponds to probability with which one can detect four photons. In order to intuitionally show the relation between $P_{\text {total }}$ with the time $\tau_{j}$, we perform a numerical simulation with the following choice of parameters: $\tau_{j}=\tau$ for all $j, \lambda_{L}=\lambda_{R}=10 \kappa$ (and $15 \kappa), \gamma_{l}=\gamma_{r}=0.1 \kappa$. See FIG. 2. One can find the maximal probability is at the time $\tau=\arctan \left(\frac{2 \Omega_{k}}{\Gamma}\right) / \Omega_{k}$. Our efficiency is close to those in other preparations of entanglement of qubits $[31,32]$. In fact, both the increasement of $\lambda_{i}$ properly and the improvement of the rate $\kappa / \gamma$ can improve the total probability, as can also be seen from FIG. 2.

The entangled state of a pair of qutrits is relatively stable against spontaneous emission because quantum information is encoded in the degenerate ground states which are not sensitive to spontaneous emission effect. In other words, once the entangled state is prepared, it has longer life. What is more, one has to require that the rotated angle $\theta$ of $F S-P B S$ satisfies $\tan ^{2}(2 \theta)=2$ and $\lambda_{L}=\lambda_{R}$ in order to obtain the maximally entangled qutrits, otherwise the fidelity will be reduced. In fact, if $\tan ^{2}(2 \theta)=2.5$, one can find that the fidelity of the final state $|\Psi(\theta)\rangle$ is $F=\left|\langle\Psi(\theta) \mid \Psi\rangle_{A B}\right|^{2} \simeq 0.99$; If $\frac{\lambda_{L}}{\lambda_{R}}=1.1$, the fidelity $F$ of the final state $\left|\Psi^{\prime}\right\rangle$ is $F=\left|\left\langle\Psi^{\prime} \mid \Psi\right\rangle_{A B}\right|^{2} \simeq 0.98$. Both show slight influences. What is more, the inefficient detections leading to less clicks of the detectors only reduce the success probability instead of fidelity, so does the failure of initialization of the initial states of atoms and cavities. As mentioned in Ref. [25], because the photons from spontaneous emissions to free modes run with random directions, they can not be registered by the detectors. Thus the fidelity is not influenced too. The reduction of the success probability has been included in our result.

In fact, one can find that the same conclusion can be drawn if one takes the coincidence detections. It is also worthy of being noted that if Alice and Bob trap the atoms at their hands in a single cavity, respectively, the same result can also be obtained so long as the detections of photons can not provide any information on which atom a photon is emitted from. In this way, the entangled two qutrits are trapped in a single cavity respectively which might be not only more convenient to the further operations of a single qutrit in the quantum information processing later but it is not necessary to control 4 cavities in practice. In this way, one can also avoid the simultaneous preparation four identical cavites+atoms in practice. Of course, if the four (or two) systems of cavity+atom are not identical, one has to analyze the fidelity of the final state.

What we used also consists of linear optical elements, and photon detectors, which has been widely used to entangle photons. In particular, the similar optical setups has been used to successfully prepare $\mathrm{W}$ states of photons 
in experiment [35]. Therefore, our schemes are feasible by current technologies.

In summary, we have presented a simple scheme to entangle two distant qutrits only using linear optical elements. The key is the indistinguishability of photons emitted from the entangled atoms. Since the quantum information is encoded on the degenerate ground states of a pair of atoms, the entanglement of the two qutrits is relatively stable. Our scheme has been shown to be suitable for both cases with and without coincidence detections. However, the latter can dramatically relax the conditions of the experimental realization. The scheme allows the two atoms at Alice's or Bob's side trapped in a single cavity which might be convenient to further applications. It has been shown that the fidelity is independent of the inefficient detections, spontaneous emissions and so on.

This work was supported by the National Natural Science Foundation of China, under Grant No. 10747112, No. 10575017 and No. 60578014.

\section{References}

1. S. Bell, Physics (Long Island City, N. Y.) 1, 195 (1964).

2. C. H. Bennett and D. P. Divincenzo, Nature (London) 404, 247 (2000).

3. J. I. Cirac and P. Zoller, Phys. Rev. Lett. 74, 4091 (1995).

4. E. Jané, M. B. Plenio, and D. Jonathan, Phys. Rev. A 65, 050302(R) (2002).

5. Manzoor Ikram and Farhan Saif, Phys. Rev. A 66, 014304 (2002).
6. Shi-Biao Zheng, Phys. Rev. A 65, 051804(R) (2002); ShiBiao Zheng, ibid. 66, 060303(R) (2002).

7. C. A. Sackett, D. Kielpinski et al., Nature (London) 404, $256(2000)$

8. C. Monroe, D. M. Meekhof, B. E. King et al., Phys. Rev. Lett. 75, 4714 (1995).

9. D. Bouwmeester, J.-W. Pan, M. Daniell et al., Phys. Rev. Lett. 82, 1345 (1999); J.-W. Pan et al., ibid. 86, 4435 (2001); J.-W. Pan et al., Nature (London) 403, 515 (2000).

10. Sixia Yu and Chang-pu Sun, Phys. Rev. A 61, 022310 (2000).

11. C. H. Bennett, D. P. DiVincenzo, P. W. Shor et al., Phys. Rev. Lett. 87, 077902 (2001).

12. P. Agrawal, P. Parashar, and A. K. Pati, quant-ph/0304006.

13. H. Bechmann-Pasquinucci and A. Peres, Phys. Rev. Lett. 85, $3313(2000)$.

14. D. Bru $\beta$ and C. Macchiavello, Phys. Rev. Lett. 88, 127901 (2002).

15. N. J. Cerf, M. Bourennane, A. Karrlson, and N. Gisin, Phys. Rev. Lett. 88, 127902 (2002);.

16. Dagomir Kaszlikowski et al., e-print quant-ph/0206170 Thomas Durt et al., e-print quant-ph/0207057.

17. Gilad Gour, Phys. Rev. A 70, 042301 (2004).

18. X. B. Zou and W. Mathis, Phys. Rev. A 70, 035802 (2004).

19. Guang-Sheng Jin, Shu-Shen Li, Song-Lin Feng et al., Phys. Rev. A 71, 034307 (2005).

20. Asoka Biswas, G. S. Agarwal, J. Mod. Opt. 51, 1627 (2004).

21. R. T. Thew, A. Acin, H. Zbinden et al. , quant-ph/0307122. 
22. Li-Xiang Cen, Bang-Pin Hou, Ming-Lun Chen, quant-ph/0608105.

23. Chang-shui Yu et al., Phys. Rev. A 75, 044301 (2007).

24. Xun-Li Feng et al., Phys. Rev. Lett. 90, 217902 (2003).

25. C. Cabrillo et al., Phys. Rev. A 59, 1025 (1999).

26. S. Bose et al., Phys. Rev. Lett. 83, 5158 (1999); C. Simon et al., ibid. 91, 110405 (2003).

27. X. B. Zou et al., Phys. Rev. A 66, 044302 (2002); X. B. Zou et al., ibid. 68, 024302 (2003); X. B. Zou et al., ibid. 69, 052314 (2004).

28. C. D. Fidio et al., J. Opt. B: Quantum Semiclass. Opt. 5, 105 (2003).

29. L. -M. Duan et al., Phys. Rev. Lett. 90, 253601 (2003).

30. M. B. Plenio and P. L. Knight, Rev. Mod. Phys. 70, 101 (1998).

31. T. Yamamoto et al., Phys. Rev. A 66, 064301 (2002).

32. H. F. Hofmann and S. Takeuchi, Phys. Rev. Lett. 88, 147901 (2002).

33. W. Lange and H. J. Kimble, Phys. Rev. A 61, 063817 (2000).

34. J. Hong and H. -W. Lee, Phys. Rev. Lett. 89, 237901 (2002).

35. M. Eibl et al., Phys. Rev. Lett. 92, 077901 (2004). 